\title{
Primitive angiosperms in the Indian Subcontinent: taxonomic diversity and geographical distribution of Magnoliaceae Juss. (APG IV)
}

\author{
Uma Shankar \\ Department of Botany, North-Eastern Hill University, Shillong 793 022, Meghalaya, India \\ E-mail: arshuma@yahoo.com
}

[Received 12.05.2020; Revised 24.06.2020; Accepted 27.06.2020; Published 30.06.2020]

\begin{abstract}
Some new insights in patterns of diversity and distribution of primitive angiospermic group led by the family Magnoliaceae in the Indian Subcontinent are presented in this article. The Subcontinent harbours nearly $10 \%$ of accepted taxa of Magnoliaceae in the world. All native taxa are restricted to the eastern region of the Subcontinent except one, Magnolia nilagirica, which is endemic to the Western Ghats-Sri Lanka global hotspot of biodiversity. All the remaining 24 native taxa occur in the northeastern region of India. Some of these radiate into Bangladesh, Bhutan and Nepal. About one-fourth of these taxa are endemic to India. $M$. pleiocarpa is endemic to Assam and $M$. punduana is endemic to Meghalaya. While only one species, $M$. cathcartii, is of temperate environment, four species are subtropical to temperate ( $M$. campbellii, $M$. doltsopa, M. kisopa, M. lanuginosa), 9 species of predominantly subtropical and 11 species of low montane or tropical environment. All native species are of phanerophytic life-form, i.e., trees of various sizes, except one shrub, $M$. globosa. Seven introduced and two hybrid taxa also occur in the Subcontinent and of these, southern magnolia, M. grandiflora, is the most popular and widespread in gardens and plantations. The horse-shoe shaped mountainous terrain of the northeast India is a centre of diversity of magnolias and supposedly represents a subset of "Southeast Asian" centre of origin and diversification of this primitive angiosperm group.
\end{abstract}

Key words: Magnoliids, Magnoliaceae, Magnolias, India Subcontinent, Primitive angiosperms.

\section{INTRODUCTION}

The APG system of classification recognized Magnoliids as a clade of primitive angiosperms comprising Magnoliales, Laurales, Piperales and Canellales (APG IV 2016). The Order Magnoliales comprises six families, viz., Annonaceae, Degeneriaceae, Eupomatiaceae, Himantandraceae, Magnoliaceae, and Myristicaceae. Of these, Magnoliaceae comprises only two genera, viz., Liriodendron L. and Magnolia Plum. ex L. Earlier, a number of genera, such as, Aromadendron, Michelia, Manglietia, Paramichelia, Sampacca, Talauma, had been named in Magnoliaceae, but nomenclatural changes have merged them into a single genus Magnolia. This has rendered the already published regional floras of limited use in terms of ready availability of accepted binomials and respective synonyms, especially to the students, research scholars, ecologists and foresters in confusion.

Plant taxonomists regard Magnoliaceae as one of the most primitive angiosperm families due to its presence in early fossil records, gymnosperm-like pollen (with one aperture for germination), wood anatomy and flower structure. Some important diagnostic characteristics of Magnoliaceae are as follows: The leaves are simple in alternate or spiral arrangement. Large deciduous furry stipules generally leave scars around young branches. The bisexual, radially symmetrical flowers are large eccentric and solitary. The receptacle is swollen which hosts numerous spirally arranged floral appendages (no sign of a reduction in parts). The sepals and 


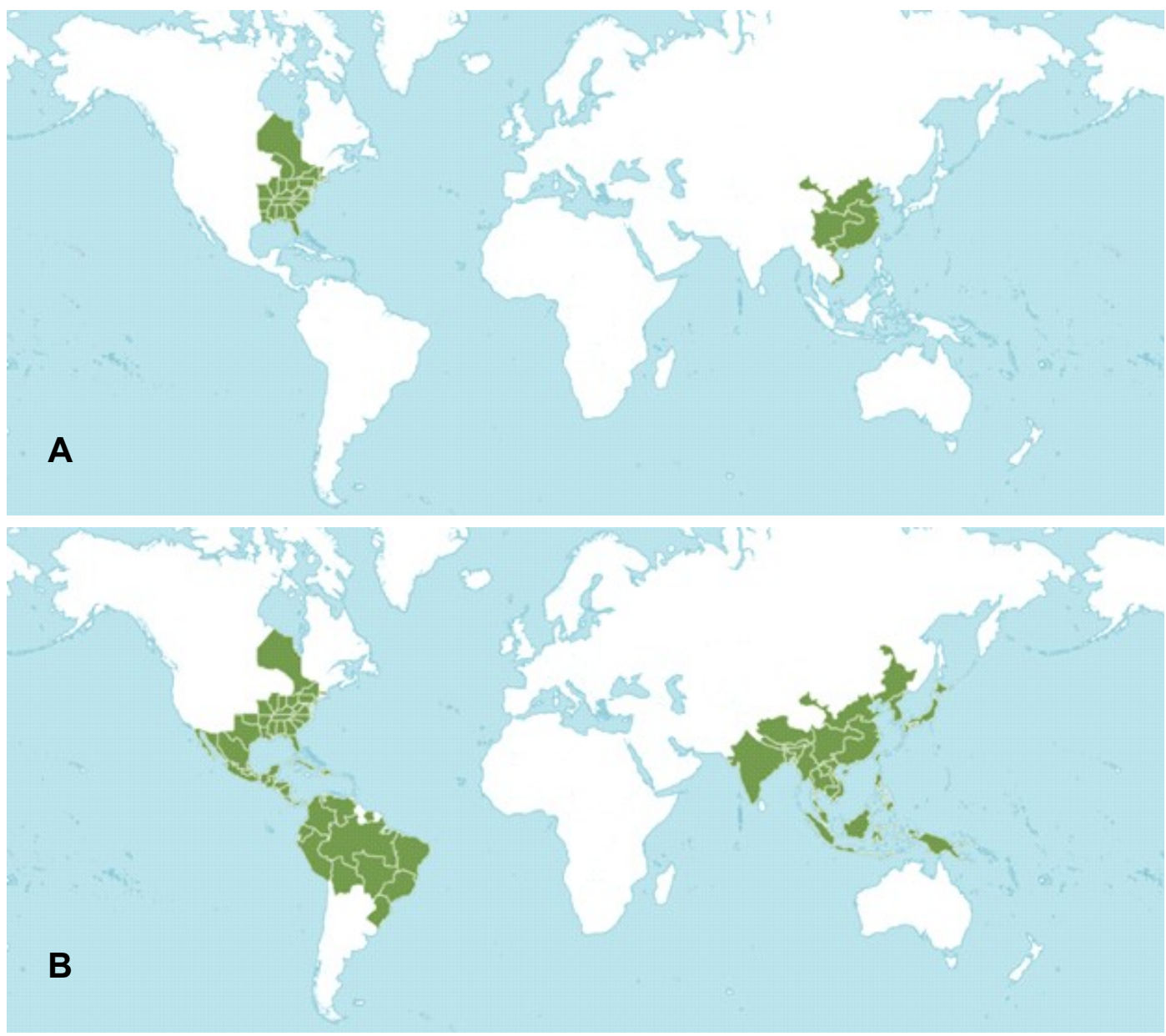

Figure 1. Global distribution of genus: A. Liriodendron L.; and B. Magnolia Plum. ex L. as per Kew Science (POWO 2020)

petals are indistinguishable (often referred to as tepals). The anthers are undifferentiated from filaments. Aggregated follicles make up the cone-shaped fruit structure which house brightly coloured funiculate seeds (Every \& Baracat 2009).

It is revealed that the reconstruction of the first angiospermic flower from the fossil records, 250 to 140 million years old, looked like a magnolia (Sauquet et al. 2017). Nonetheless, the origin of the angiosperm flower remains among the most difficult and most important unresolved topics in evolutionary biology (Frohlich \& Chase 2007; Friis et al. 2011; Doyle 2012, 2014). The extant or living magnolias show classical 'disjunct distribution' in two distant geographies, vi\%., the Americas (between Canada and Brazil in the west), and the Southeast Asia (predominantly in Indo-Malayan ecoregion in the East), and surprisingly nowhere in between (Figure 1). This has been a puzzle amongst the botanists for nearly two centuries, although some explanations for this intriguing distribution have been offered (Nooteboom 2011). Magnolias present an excellent example of allopatric speciation, according to which the new species are formed as a result of geographic isolation and new selection pressures (SanchezVelasquez et al. 2016). There has been a debate which of the two centres of the distribution is actually a centre of origin of magnolias and the conclusion that "neither of the present centres of diversity is the centre of origin" prevails currently (Nooteboom 2011). 
In Indian Subcontinent, the habit of magnolias varies from shrub to large tree and none of the species is a climber. Only a subset of all magnolias found in Southeast Asia is available in Indian Subcontinent and most of it occurs in the Northeastern region. Kundu (2009) enlisted 51 taxa from Indian subcontinent but including Magnoliaceae Juss. (s.s.), Illiciaceae A.C.Sm. and Schisandraceae Blume in a preliminary checklist following old nomenclature. Khuraijam \& Goel (2015) presented a reclassification of species of Magnoliaceae from India alone. This article presents an updated account of the taxonomic diversity of Magnoliaceae following APG-IV nomenclature, geographical distribution following Kew Science (POWO 2020) and conservation status of Magnoliaceae taxa (Global Tree Specialist Group 2014) that are native to or introduced into the Indian Subcontinent along with their synonyms and country-wise distributions.

\section{MATERIAL AND METHODS}

This study focuses on members of Magnoliaceae Juss. growing in the Indian subcontinent. The collected specimens were studied in the Botanical Survey of India's herbaria viz. CAL and ASSAM and of own collection. The already published floras and reports were screened for preparing a checklist of the members of Magnoliaceae. The nomenclatural changes were taken into account and the latest spellings of the generic names, epithets and authorities were verified in Kew Science resources (The Plant List 2013; POWO 2020). The habitat, habit and life-forms were compiled from observation in specimens, published resources, if available, and from own observations. The country-wise distribution records were compiled from authentic published literature. Similarly, the distribution records for different states and territories in India were compiled from the regional floras and accessible research articles.

\section{RESULTS AND DISCUSSION}

The genus Liriodendron L. (commonly called "tulip trees") has only two species in the world: one is restricted to Southeast Asia (Liriodendron cbinense (Hemsl.) Sarg. with distribution in China North-Central, China South-Central, China Southeast, Vietnam) and the other is restricted to the Americas (Liriodendron tulipifera L. with distribution in Alabama, Arkansas, Connecticut, Delaware, District of Columbia, Florida, Georgia, Illinois, Indiana, Kentucky, Louisiana, Maryland, Masachusettes, Michigan, Mississippi, Missouri, New Jersey, New York, North Carolina, Ohio, Ontario, Pennsylvania, Rhode I., South Carolina, Tennessee, Vermont, Virginia, West Virginia) (POWO 2020). This genus is completely absent in the Indian Subcontinent (Figure 1A), except a few planted individuals of L. tulipifera in FRI campus in Dehradun and in Darjeeling. Currently, the genus Magnolia Plum. ex L. has 320 accepted species (327 taxa) in the world and is widely spread in both the centres of distribution with nearly one-half of the taxa in each (Figure 1B).

The Indian Subcontinent harbours nearly 10\% of these accepted taxa and most of them are restricted to the northeastern region of India, Central and East Himalaya, Nepal and Bhutan, and only one species, Magnolia nilagirica, is present in the Western Ghats and Sri Lanka Global Hotspot of Biodiversity. The compiled list of the accepted species of the genus Magnolia reveals 25 native, seven introduced and two hybrid taxa in the Indian Subcontinent (Table 1). The synonyms of all these accepted taxa are provided to facilitate the easy browsing and linking of the binomials available in already published floras in the region (Hooker 1872-1897; Kanjilal et al. 1934; Balakrishnan 1981-1983; Deb 1981-1983; Joseph 1982; Haridasan 1985-1987; Das \& Chanda 1987; Hajra et al. 1996; Chowdhury 2005; Giri et al. 2008; Singh et al. 2000, 2002; Barooah \& Ahmed 2014; Mao et al. 2016, 2017). 
While $M$. griffithii and $M$. grandiflora have no synonyms, M. liliifera has 49 synonyms which is the maximum number for the species inventoried from the Indian Subcontinent. M. champaca, M. gustavi, M. mannii, M. pleiocarpa, M. rabaniana, M. sirindhorniae have only one synonym each (Table 1). All magnolias native to the Indian Subcontinent are distributed between Tibet in the northwest and Malayan peninsula in the Southeast Asian centre of diversity. M. pleiocarpa (basionym: Pachylarnaxpleiocarpa Dandy) has the narrowest distribution and is endemic to only one location, i.e., Jeypore Reserve Forest in North Lakhimpur district of Assam (Figlar \& Nooteboom 2004; Global Tree Specialist Group 2014), although it is believed to occur in nearby Arunachal Pradesh (Khuraijam \& Goel 2015). M. punduana is documented as endemic to Meghalaya (Balakrishnan 1981-1983; Haridasan 1985-1987; Iralu et al. 2020), but has also been listed in the flora of Arunachal Pradesh (Hajra et al. 1996) and flora of Manipur (Singh et al. 2000). M. mannii and M. oblonga are endemic to northeast India and adjoining territories in Bangladesh. On the other hand, $M$. liliifera has the widest distribution from East Himalaya to Sulawesi (Table 1). Among the introduced species, $M$. grandiflora has the broadest occurrences due to large-scale introductions. Magnolia liliiflora is grown in courtyard of the Buddhist monastries and in botanic gardens in Sikkim and Darjeeling.

While only one native species, $M$. cathcartii, was temperate, four species were subtropical to temperate (M. campbellii, $M$. doltsopa, M. kisopa, M. lanuginosa), 9 species were subtropical and 11 species were of low montane or tropical environment (Table 1). Among introduced taxa, M. liliiflora is of temperate, M. coco and M. obovata are subtropical and M. grandiflora, M. figo var. figo, M. montana and M. sirindhorniae are of tropical or low montane environment. Two hybrid taxa, M. $\times$ alba and $M . \times$ soulangeana are cultivated in tropical regions of Asia (Table 1).

Table 1.Taxonomic diversity and nativity of Magnoliaceae in Indian Subcontinent.

\begin{tabular}{|c|c|c|}
\hline Sl. & Accepted taxa and their known synonyms & Climate and nativity \\
\hline & Native taxa & \\
\hline 1 & $\begin{array}{l}\text { Magnolia baillonii Pierre } \\
\text { 1. Aromadendron baillonii (Pierre) Craib } \\
\text { 2. Aromadendron spongocarpum (King) Craib } \\
\text { 3. Magnolia baillonii var. bailingia Sima \& H.Jiang } \\
\text { 4. Magnolia phellocarpa (King) H.J.Chowdhery \& P.Daniel } \\
\text { 5. Michelia baillonii (Pierre) Finet \& Gagnep. } \\
\text { 6. Michelia phellocarpa (King) Finet \& Gagnep. } \\
\text { 7. Paramichelia baillonii (Pierre) Hu } \\
\text { 8. Talauma phellocarpa King } \\
\text { 9. Talauma spongocarpa King }\end{array}$ & $\begin{array}{l}\text { Low montane; Indo- } \\
\text { Malaya }\end{array}$ \\
\hline 2 & $\begin{array}{l}\text { Magnolia campbellii Hook.f. \& Thomson [Campbell's Magnolia] } \\
\text { 1. Magnolia mollicomata W.W.Sm. } \\
\text { 2. Yulania campbellii (Hook.f. \& Thomson) D.L.Fu }\end{array}$ & $\begin{array}{l}\text { Subtropical to } \\
\text { Temperate; Central and } \\
\text { East Himalaya, } \\
\text { Myanmar and China }\end{array}$ \\
\hline 3 & $\begin{array}{l}\text { Magnolia cathcartii (Hook.f. \& Thomson) Noot. [Cathcart's } \\
\text { Magnolia] } \\
\text { 1. Alcimandra cathcartii (Hook.f. \& Thomson) Dandy } \\
\text { 2. Aromadendron cathcartii (Hook.f. \& Thomson) Sima \& S.G.Lu } \\
\text { 3. Michelia cathcartii Hook.f. \& Thomson } \\
\text { 4. Michelia gustavii King } \\
\text { 5. Sampacca cathcartii (Hook.f. \& Thomson) Kuntze }\end{array}$ & $\begin{array}{l}\text { Temperate; Tibet to } \\
\text { Indo-Malaya }\end{array}$ \\
\hline 4 & $\begin{array}{l}\text { Magnolia caveana (Hook.f. \& Thomson) D.C.S.Raju \& M.P.Nayar } \\
\text { 1. Magnolia tibetica V.S.Kumar } \\
\text { 2. Manglietia caveana Hook.f. \& Thomson } \\
\text { 3. Manglietia microtricha Y.W.Law } \\
\text { 4. Manglietia tenuifolia Hung T.Chang \& B.L.Chen }\end{array}$ & $\begin{array}{l}\text { Subtropical; Indo- } \\
\text { Malaya }\end{array}$ \\
\hline
\end{tabular}




\begin{tabular}{|c|c|c|}
\hline Sl. & Accepted taxa and their known synonyms & Climate and nativity \\
\hline & Native taxa & \\
\hline 5 & $\begin{array}{l}\text { Magnolia champaca (L.) Baill. ex Pierre [Champak tree] } \\
\text { 1. Michelia champaca L. }\end{array}$ & $\begin{array}{l}\text { Low montane; Indo- } \\
\text { Malaya }\end{array}$ \\
\hline 6 & $\begin{array}{l}\text { Magnolia champaca (L.) Baill. ex Pierre var. champaca } \\
\text { 1. Champaca michelia Noronha } \\
\text { 2. Magnolia membranacea P.Parm. } \\
\text { 3. Magnolia membranacea P.Parm. } \\
\text { 4. Michelia aurantiaca Wall. } \\
\text { 5. Michelia blumei Steud. } \\
\text { 6. Michelia enonymoides Burm.f. } \\
\text { 7. Michelia rheedei Wight } \\
\text { 8. Michelia rufinervis DC. } \\
\text { 9. Michelia serices Pers. } \\
\text { 10. Michelia suaveolens Pers. } \\
\text { 11. Sampacca enonymoides (Burm.f.) Kuntze } \\
\text { 12. Sampacca suaveolens (Pers.) Kuntze }\end{array}$ & $\begin{array}{l}\text { Subtropical; Tibet to } \\
\text { Indo-Malaya }\end{array}$ \\
\hline 7 & $\begin{array}{l}\text { Magnolia doltsopa (Buch.-Ham. ex DC.) Figlar [Manipur Magnolia] } \\
\text { 1. Magnolia excelsa Jacques } \\
\text { 2. Magnolia excelsa Wall. } \\
\text { 3. Michelia calcuttensis P.Parm. } \\
\text { 4. Michelia doltsopa Buch.-Ham. ex DC. } \\
\text { 5. Michelia excelsa (Wall.) Blume } \\
\text { 6. Michelia manipurensis Watt ex Brandis } \\
\text { 7. Michelia wardii Dandy } \\
\text { 8. Sampacca excelsa (Wall.) Kuntze }\end{array}$ & $\begin{array}{l}\text { Subtropical to } \\
\text { temperate; Tibet to } \\
\text { Indo-Malaya }\end{array}$ \\
\hline 8 & $\begin{array}{l}\text { Magnolia globosa Hook.f. \& Thomson } \\
\text { 1. Aromadendron griffithii (Hook.f. \& Thomson) Sima \& S.G.Lu } \\
\text { 2. Michelia griffithii (Hook.f. \& Thomson) Finet \& Gagnep. }\end{array}$ & $\begin{array}{l}\text { Subtropical; Tibet to } \\
\text { Indo-Malaya }\end{array}$ \\
\hline 9 & Magnolia griffithii Hook.f. \& Thomson [Griffith's Magnolia] & $\begin{array}{l}\text { Subtropical; Assam, } \\
\text { Bangladesh, Myanmar }\end{array}$ \\
\hline 10 & $\begin{array}{l}\text { Magnolia gustavi King [Gustav's Magnolia] } \\
\text { 1. Aromadendron gustavi (King) Sima \& S.G.Lu }\end{array}$ & $\begin{array}{l}\text { Low montane; Assam, } \\
\text { Myanmar, Thailand }\end{array}$ \\
\hline 11 & $\begin{array}{l}\text { Magnolia hodgsonii (Hook.f. \& Thomson) H.Keng [Himalayan } \\
\text { Magnolia] } \\
\text { 1. Lirianthe hodgsonii (Hook.f. \& Thomson) Sima \& S.G.Lu } \\
\text { 2. Talauma hodgsonii Hook.f. \& Thomson }\end{array}$ & $\begin{array}{l}\text { Low montane; Tibet to } \\
\text { Indo-Malaya }\end{array}$ \\
\hline 12 & $\begin{array}{l}\text { Magnolia hookeri (Cubitt \& W.W.Sm.) D.C.S.Raju \& M.P.Nayar } \\
\text { 1. Magnolia hookeri var. longirostrata D.X.Li \& R.Z.Zhou ex } \\
\text { X.M.Hu, Q.W.Zeng \& L.Fu } \\
\text { 2. Manglietia hookeri Cubitt \& W.W.Sm. }\end{array}$ & $\begin{array}{l}\text { Low montane; Indo- } \\
\text { Malaya }\end{array}$ \\
\hline 13 & $\begin{array}{l}\text { Magnolia insignis Wall. } \\
\text { 1. Magnolia insignis var. angustifolia (Hook.f. \& Thomson) } \\
\text { H.J.Chowdhery \& P.Daniel } \\
\text { 2. Magnolia insignis var. latifolia (Hook.f. \& Thomson) } \\
\text { H.J.Chowdhery \& P.Daniel } \\
\text { 3. Magnolia shangaensis Hu } \\
\text { 4. Manglietia insignis (Wall.) Blume } \\
\text { 5. Manglietia maguanica Hung T.Chang \& B.L.Chen } \\
\text { 6. Manglietia rufisyncarpa Y.W.Law, R.Z.Zhou \& F.G.Wang } \\
\text { 7. Manglietia yunnanensis Hu }\end{array}$ & $\begin{array}{l}\text { Low montane; Tibet to } \\
\text { Indo-Malaya }\end{array}$ \\
\hline 14 & $\begin{array}{l}\text { Magnolia kingii (Dandy) Figlar } \\
\text { 1. Michelia glabra P.Parm. } \\
\text { 2. Michelia kingii Dandy }\end{array}$ & $\begin{array}{l}\text { Subtropical; Assam, } \\
\text { Bangladesh, Bhutan }\end{array}$ \\
\hline
\end{tabular}




\begin{tabular}{|c|c|c|}
\hline Sl. & Accepted taxa and their known synonyms & Climate and nativity \\
\hline & Native taxa & \\
\hline 15 & $\begin{array}{l}\text { Magnolia kisopa (Buch.-Ham. ex DC.) Figlar [Kisopa Magnolia] } \\
\text { 1. Michelia doltsopa subsp. kisopa (Buch.-Ham. ex DC.) J.Li } \\
\text { 2. Michelia kisopa Buch.-Ham. ex DC. } \\
\text { 3. Sampacca kisopa (Buch.-Ham. ex DC.) Kuntze }\end{array}$ & $\begin{array}{l}\text { Subtropical to } \\
\text { temperate; India, } \\
\text { Nepal, Tibet }\end{array}$ \\
\hline 16 & $\begin{array}{l}\text { Magnolia lanuginosa (Wall.) Figlar \& Noot. } \\
\text { 1. Magnolia velutina (DC.) Figlar } \\
\text { 2. Michelia lanceolata E.H.Wilson } \\
\text { 3. Michelia lanuginosa Wall. } \\
\text { 4. Michelia velutina DC. } \\
\text { 5. Sampacca lanuginosa (Wall.) Kuntze }\end{array}$ & $\begin{array}{l}\text { Subtropical to } \\
\text { temperate; India, } \\
\text { Nepal, Tibet, China }\end{array}$ \\
\hline 17 & 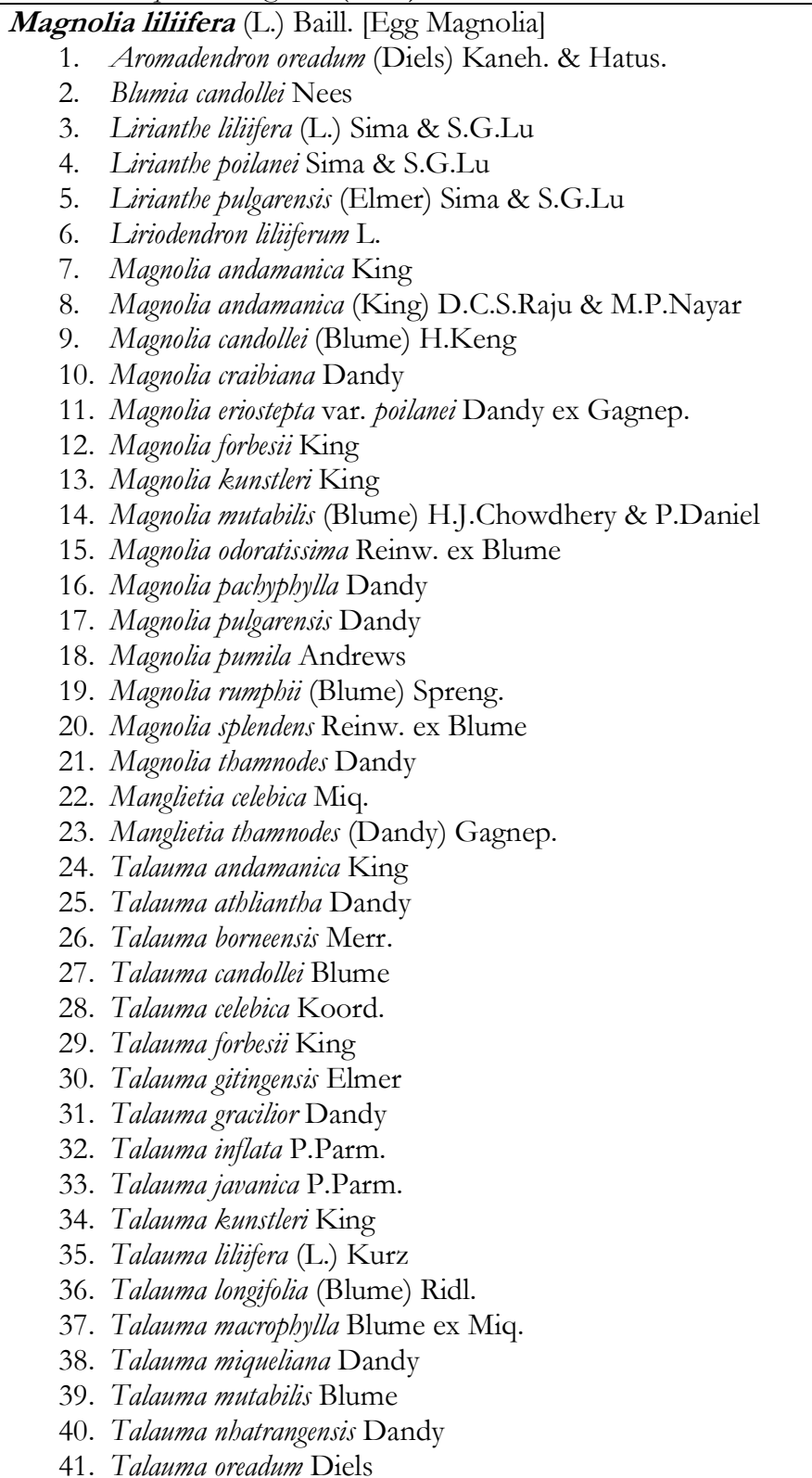 & $\begin{array}{l}\text { Low montane; East } \\
\text { Himalaya to Sulawesi }\end{array}$ \\
\hline
\end{tabular}




\begin{tabular}{|c|c|c|}
\hline Sl. & Accepted taxa and their known synonyms & Climate and nativity \\
\hline & Native taxa & \\
\hline 17 & 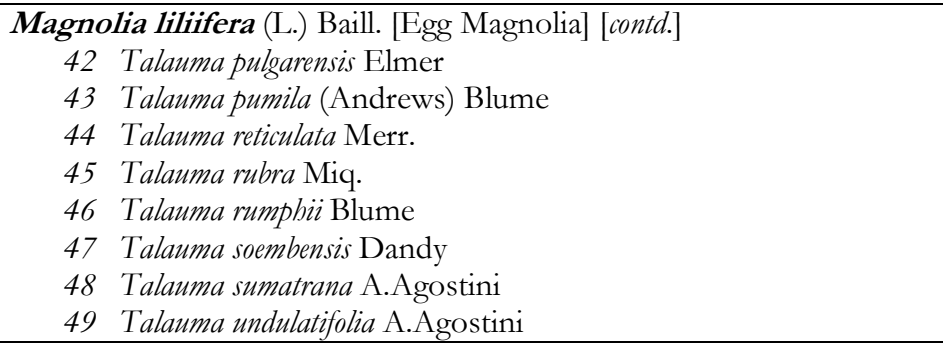 & $\begin{array}{l}\text { Low montane; East } \\
\text { Himalaya to Sulawesi }\end{array}$ \\
\hline 18 & $\begin{array}{l}\text { Magnolia mannii (King) Figlar } \\
\text { 1. Michelia mannii King }\end{array}$ & $\begin{array}{l}\text { Low montane; Assam, } \\
\text { Bangladesh }\end{array}$ \\
\hline 19 & $\begin{array}{l}\text { Magnolia nilagirica (Zenker) Figlar [Nilgiri Magnolia] } \\
\text { 1. Michelia glauca Wight } \\
\text { 2. Michelia nilagirica Zenker } \\
\text { 3. Michelia ovalifolia Wight } \\
\text { 4. Michelia pulneyensis Wight } \\
\text { 5. Michelia walkeri Wight } \\
\text { 6. Sampacca nilagirica (Zenker) Kuntze }\end{array}$ & $\begin{array}{l}\text { Low montane; India, } \\
\text { Sri Lanka }\end{array}$ \\
\hline 20 & $\begin{array}{l}\text { Magnolia oblonga (Wall. ex Hook.f. \& Thomson) Figlar } \\
\text { 1. Michelia lactea Buch.-Ham. ex Wall. } \\
\text { 2. Michelia oblonga Wall. ex Hook.f. \& Thomson } \\
\text { 3. Sampacca oblonga (Wall. ex Hook.f. \& Thomson) Kuntze }\end{array}$ & $\begin{array}{l}\text { Subtropical; Assam, } \\
\text { Bangladesh }\end{array}$ \\
\hline 21 & $\begin{array}{l}\text { Magnolia pealiana King } \\
\text { 1. Aromadendron pealianum (King) Sima \& S.G.Lu } \\
\text { 2. Michelia pealiana (King) Finet \& Gagnep. }\end{array}$ & $\begin{array}{l}\text { Low montane; Assam } \\
\text { (Jeypore Reserve } \\
\text { Forest), Endemic }\end{array}$ \\
\hline 22 & $\begin{array}{l}\text { Magnolia pleiocarpa (Dandy) Figlar \& Noot. } \\
\text { 1. Pachylarnax pleiocarpa Dandy }\end{array}$ & $\begin{array}{l}\text { Subtropical; Endemic } \\
\text { to Lakhimpur in Assam }\end{array}$ \\
\hline 23 & $\begin{array}{l}\text { Magnolia pterocarpa Roxb. } \\
\text { 1. Lirianthe grandiflora Spach } \\
\text { 2. Lirianthe pterocarpa (Roxb.) Sima \& S.G.Lu } \\
\text { 3. Liriodendron grandiflorum Roxb. } \\
\text { 4. Liriodendron indicum Spreng. } \\
\text { 5. Magnolia sphenocarpa Hook.f. \& Thomson } \\
\text { 6. Michelia macrophylla D.Don } \\
\text { 7. Sphenocarpus pterocarpus (Roxb.) K.Koch } \\
\text { 8. Talauma roxburghii G.Don }\end{array}$ & $\begin{array}{l}\text { Subtropical; Central } \\
\text { Himalaya to } \\
\text { Bangladesh }\end{array}$ \\
\hline 24 & $\begin{array}{l}\text { Magnolia punduana (Hook.f. \& Thomson) Figlar [Pandua } \\
\text { Magnolia] } \\
\text { 1. Michelia punduana Hook.f. \& Thomson } \\
\text { 2. Sampacca punduana (Hook.f. \& Thomson) Kuntze }\end{array}$ & $\begin{array}{l}\text { Subtropical; Endemic } \\
\text { to Meghalaya and } \\
\text { Arunachal Pradesh }\end{array}$ \\
\hline \multirow[t]{2}{*}{25} & $\begin{array}{l}\text { Magnolia rabaniana (Hook.f. \& Thomson) D.C.S.Raju \& M.P.Nayar } \\
\text { 1. Talauma rabaniana Hook.f. \& Thomson }\end{array}$ & $\begin{array}{l}\text { Low montane; NE } \\
\text { India, Myanmar }\end{array}$ \\
\hline & Introduced and hybrid taxa & \\
\hline 26 & $\begin{array}{l}\text { Magnolia } \boldsymbol{x} \text { alba (DC.) Figlar [White Champaca; hybrid of } M . \\
\text { champaca and } M . \text { montana] } \\
\text { 1. Michelia } \times \text { alba } \text { DC. } \\
\text { 2. Michelia } \times \text { longifolia Blume } \\
\text { 3. Sampacca } \times \text { longifolia (Blume) Kuntze }\end{array}$ & $\begin{array}{l}\text { Cultivated in tropical } \\
\text { regions of East Asia; } \\
\text { origin uncertain }\end{array}$ \\
\hline 27 & $\begin{array}{l}\text { Magnolia coco (Lour.) DC. [Dwarf or Coconut Magnolia] } \\
\text { 1. Lirianthe coco (Lour.) N.H.Xia \& C.Y.Wu } \\
\text { 2. Liriodendron coco Lour. } \\
\text { 3. Liriopsis pumila Spach ex Baill. } \\
\text { 4. Talauma coco (Lour.) Merr. }\end{array}$ & $\begin{array}{l}\text { Subtropical; S. China to } \\
\text { N. Vietnam, Taiwan }\end{array}$ \\
\hline
\end{tabular}


144 Diversity and distribution of Magnoliaceae

\begin{tabular}{|c|c|c|}
\hline Sl. & Accepted taxa and their known synonyms & Climate and nativity \\
\hline & Native taxa & \\
\hline 28 & Magnolia grandiflora L. [Southern Magnolia] & $\begin{array}{l}\text { Low montane to } \\
\text { Subtropical; USA }\end{array}$ \\
\hline 29 & $\begin{array}{l}\text { Magnolia figo (Lour.) DC. var. figo [Banana shrub] } \\
\text { 1. Liriodendron figo Lour. } \\
\text { 2. Michelia figo (Lour.) Spreng. }\end{array}$ & $\begin{array}{l}\text { Low montane; China, } \\
\text { Korea }\end{array}$ \\
\hline 30 & $\begin{array}{l}\text { Magnolia liliiflora Desr. [Lily Magnolia] } \\
\text { 1. Lassonia quinquepeta Buc'hoz } \\
\text { 2. Magnolia atropurpurea Steud. } \\
\text { 3. Magnolia discolor Vent. } \\
\text { 4. Magnolia gracilis Salisb. } \\
\text { 5. Magnolia liliiflora f. nigra (G.Nicholson) Geerinck } \\
\text { 6. Magnolia plena C.L.Peng \& L.H.Yan } \\
\text { 7. Magnolia polytepala Y.W.Law, R.Z.Zhou \& R.J.Zhang } \\
\text { 8. Magnolia purpurea Curtis } \\
\text { 9. Magnolia quinquepeta (Buc'hoz) Dandy } \\
\text { 10. Talauma sieboldii Miq. } \\
\text { 11. Yulania japonica Spach } \\
\text { 12. Yulania liliiflora (Desr.) D.L.Fu }\end{array}$ & $\begin{array}{l}\text { Warm to cold } \\
\text { temperate; China }\end{array}$ \\
\hline 31 & $\begin{array}{l}\text { Magnolia montana (Blume) Figlar [Japanese cucumber tree] } \\
\text { 1. Michelia ecicatrisata Miq. } \\
\text { 2. Michelia montana Blume } \\
\text { 3. Sampacca montana (Blume) Kuntze }\end{array}$ & $\begin{array}{l}\text { Low montane; } \mathrm{W} . \\
\text { Malesia to Lesser } \\
\text { Sundalands (Bali) }\end{array}$ \\
\hline 32 & $\begin{array}{l}\text { Magnolia obovata Thunb. } \\
\text { 1. Houpoea obovata (Thunb.) N.H.Xia \& C.Y.Wu } \\
\text { 2. Magnolia glanca Thunb. } \\
\text { 3. Magnolia hirsuta Thunb. } \\
\text { 4. Magnolia honogi P.Parm. } \\
\text { 5. Magnolia hoonoki Siebold } \\
\text { 6. Magnolia bypoleuca Siebold \& Zucc. }\end{array}$ & Subtropical; Japan \\
\hline 33 & $\begin{array}{l}\text { Magnolia sirindhorniae Noot. \& Chalermglin } \\
\text { 1. Michelia sirindhorniae (Noot. \& Chalermglin) N.H.Xia \& } \\
\text { X.H.Zhang }\end{array}$ & $\begin{array}{l}\text { Tropical or low } \\
\text { montane; Thailand }\end{array}$ \\
\hline 34 & $\begin{array}{l}\text { Magnolia } \times \text { soulangeana Soul.-Bod. [Saucer Magnolia; hybrid of } \\
\text { M. denudata and } M . \text { liliiflora }] \\
\text { 1. Magnolia } \times \text { brozzonii Millais } \\
\text { 2. Magnolia } \times \text { lenneana }(\text { Lem.) Koehne } \\
\text { 3. Magnolia } \times \text { lennei } \text { Van Houtte } \\
\text { 4. Yulania } \times \text { lenneana Lem. } \\
\text { 5. Yulania } \times \text { soulangeana } \text { (Soul.-Bod.) D.L.Fu }\end{array}$ & $\begin{array}{l}\text { Cultivated in tropical } \\
\text { regions of East Asia; } \\
\text { South Central China to } \\
\text { N. Vietnam, Taiwan }\end{array}$ \\
\hline
\end{tabular}

The magnolias occur in phanerophytic life-forms, i.e., shrubs or trees of various sizes. The herbs and climbers are unknown. In Indian Subcontinent, all native species are trees of various sizes barring only one species, viz., Magnolia globosa, which is a shrub (Table 2). Of the remaining 24 native species, five are small to intermediate trees, seven are intermediate-sized trees, six are intermediate to large trees, and six are large trees. Among introduced magnolias in the region, two are large trees, two are intermediate trees and one is a shrub. Of 25 native species, only six are deciduous and the remaining 19 species are evergreen. Among introduced magnolias in the region, five are evergreen and two are deciduous (Table 2). Of two hybrid taxa, one is evergreen and one is deciduous. Cicuzza et al. (2007) estimated that $22 \%$ of magnolias in the world are critically endangered (CR), 41\% endangered (EN), 16\% vulnerable (VU), 5\% near threatened (NT), $6 \%$ of least concern (LC), about $6 \%$ have insufficient data (DD) and 
about 94 species are not evaluated (NE). The conservation status of nearly one-half of species in the Indian Subcontinent is known as per IUCN system (Table 2). M. pleiocarpa and $M$. sirindhorniae are critically endangered, $M$. gustavi and $M$. pealiana are endangered, $M$. mannii, $M$. nilagirica and $M$. punduana are vulnerable, and M. campbellii, M. globosa, M. hodgsonii, $M$. insignis, $M$. liliifera and M. grandiflora are of least concern. Three species are data deficient and 18 taxa are yet to be evaluated. Almost all native species are in their habitats in the subcontinent.

Table 2. Life-form (habit), phenology and threatened status of Magnoliaceae taxa found in Indian subcontinent.

\begin{tabular}{|c|c|c|c|}
\hline Taxon name & Life-form/ Habit & Phenology & $\begin{array}{c}\text { Threatened } \\
\text { status }\end{array}$ \\
\hline \multicolumn{4}{|l|}{ Native taxa } \\
\hline 1. Magnolia baillonii & Large tree & Evergreen & $\mathrm{NE}$ \\
\hline 2. Magnolia campbellii & Intermediate to large tree & Deciduous & LC \\
\hline 3. Magnolia cathcartii & Large tree & Evergreen & $\mathrm{NE}$ \\
\hline 4. Magnolia caveana & Intermediate tree & Evergreen & $\mathrm{NE}$ \\
\hline 5. Magnolia champaca & Intermediate to large tree & Evergreen & $\mathrm{NE}$ \\
\hline 6.Magnolia champaca var. champaca & Large tree & Evergreen & $\mathrm{NE}$ \\
\hline 7. Magnolia doltsopa & Small to intermediate tree & Deciduous & $\mathrm{NE}$ \\
\hline 8. Magnolia globosa & Shrub & Deciduous & LC \\
\hline 9. Magnolia griffithii & Intermediate to large tree & Evergreen & DD \\
\hline 10. Magnolia gustavi & Large tree & Evergreen & EN B1ab(i,iii) \\
\hline 11. Magnolia hodgsonii & Small to intermediate tree & Evergreen & $\mathrm{LC}$ \\
\hline 12. Magnolia hookeri & Intermediate to large tree & Evergreen & $\mathrm{NE}$ \\
\hline 13. Magnolia insignis & Intermediate to large tree & Evergreen & $\mathrm{LC}$ \\
\hline 14. Magnolia kingii & Intermediate tree & Evergreen & $\mathrm{NE}$ \\
\hline 15. Magnolia kisopa & Intermediate tree & Deciduous & $\mathrm{NE}$ \\
\hline 16. Magnolia lanuginosa & Intermediate to large tree & Deciduous & $\mathrm{NE}$ \\
\hline 17. Magnolia liliifera & Intermediate tree & Evergreen & LC \\
\hline 18. Magnolia mannii & Intermediate tree & Evergreen & VU B1ab(i,iii) \\
\hline 19. Magnolia nilagirica & Intermediate tree & Evergreen & VU B1ab(i,iii) \\
\hline 20. Magnolia oblonga & Large tree & Evergreen & $\mathrm{NE}$ \\
\hline 21. Magnolia pealiana & Small to intermediate tree & Evergreen & EN B1ab(i,iii) \\
\hline 22. Magnolia pleiocarpa & Large tree & Evergreen & CR B1ab(i,iii) \\
\hline 23. Magnolia pterocarpa & Small to intermediate tree & Deciduous & $\mathrm{NE}$ \\
\hline 24. Magnolia punduana & Small to intermediate tree & Evergreen & VU B1ab(i,iii) \\
\hline 25. Magnolia rabaniana & Intermediate tree & Evergreen & DD \\
\hline \multicolumn{4}{|l|}{ Introduced and hybrid taxa } \\
\hline 26. Magnolia $\times$ alba & Intermediate tree & Evergreen & $\mathrm{NE}$ \\
\hline 27. Magnolia coco & Shrub & Evergreen & $\mathrm{NE}$ \\
\hline 28. Magnolia grandiflora & Large tree & Evergreen & LC \\
\hline 29. Magnolia figo var. figo & Shrub & Evergreen & $\mathrm{NE}$ \\
\hline 30. Magnolia liliiflora & Shrub to small tree & Deciduous & $\mathrm{DD}$ \\
\hline 31. Magnolia montana & Large tree & Evergreen & $\mathrm{NE}$ \\
\hline 32.Magnolia obovata & Intermediate tree & Deciduous & $\mathrm{NE}$ \\
\hline 33.Magnolia sirindhorniae & Intermediate tree & Evergreen & CR B1ab(i) \\
\hline 34. Magnolia $\times$ soulangeana & Intermediate tree & Deciduous & $\mathrm{NE}$ \\
\hline
\end{tabular}




\section{Diversity and distribution of Magnoliaceae}

Of seven countries in the Indian Subcontinent, the magnolias occur in Nepal, Bhutan, India, Bangladesh and Sri Lanka, and are absent in Pakistan and Maldives (Table 3). The maximum number of taxa occur in India (34 species) followed by Bhutan (13 species), Bangladesh (10 species) and Nepal (10 species). The ranges of the Western Ghats-Sri Lanka global hotspot of biodiversity harbour only one species, $M$. nilagirica. This species has been claimed to occur in Assam (Barooah \& Ahmed 2014) which is doubtful and needs affirmation. The remaining 24 native species occur in Central Himalaya (Nepal), East Himalaya (Sikkim, Bhutan and Arunachal Pradesh), and northeastern region of India (Assam, Manipur, Meghalaya, Mizoram, Nagaland, and Tripura). Not a single species occurs in all five countries in the Subcontinent (Table 3). Three species, viz., M. doltsopa, M. hodgsonii and M. pterocarpa occur in four countries. Of species found in Nepal, all are shared with India and nine species with Bhutan (except $M$. insignis). Bhutan shares all its species with India and only four species with Bangladesh ( $M$. doltsopa, $M$. hodgsonii, $M$. kingii, $M$. pterocarpa). Bangladesh shares all its species with India (Table 3). Succinctly, the northeastern region of India is the centre of diversification of magnolias in the Indian Subcontinent and is a subset of "Southeast Asian" centre of origin and diversification of this primitive angiosperm group. A total of six species (M. caveana, M. gustavi, M. hookeri, M. pealiana, M. pleiocarpa, and M. punduana) are endemic to India if only political boundaries are to be considered (Table 3).

Table 3. Country-wise distribution of Magnoliaceae taxa in the Indian Subcontinent.

\begin{tabular}{|l|c|c|c|c|c|}
\hline Taxon name & Nepal & Bhutan & India & Bangladesh & $\begin{array}{c}\text { Sri } \\
\text { Lanka }\end{array}$ \\
\hline Native taxa & & & & & \\
\hline 1. Magnolia baillonii & & & $\mathrm{P}$ & $\mathrm{P}$ & \\
\hline 2. Magnolia campbellii & $\mathrm{P}$ & $\mathrm{P}$ & $\mathrm{P}$ & & \\
\hline 3. Magnolia cathcartii & & $\mathrm{P}$ & $\mathrm{P}$ & & \\
\hline 4. Magnolia caveana & & & $\mathrm{P}$ & & \\
\hline 5. Magnolia champaca & & & $\mathrm{P}$ & $\mathrm{P}$ & \\
\hline 6.Magnolia champaca var. champaca & & & $\mathrm{P}$ & $\mathrm{P}$ & \\
\hline 7. Magnolia doltsopa & $\mathrm{P}$ & $\mathrm{P}$ & $\mathrm{P}$ & $\mathrm{P}$ & \\
\hline 8. Magnolia globosa & $\mathrm{P}$ & $\mathrm{P}$ & $\mathrm{P}$ & & \\
\hline 9. Magnolia griffitbii & & & $\mathrm{P}$ & $\mathrm{P}$ & \\
\hline 10. Magnolia gustavi & & & $\mathrm{P}$ & & \\
\hline 11. Magnolia hodgsonii & $\mathrm{P}$ & $\mathrm{P}$ & $\mathrm{P}$ & $\mathrm{P}$ & \\
\hline 12. Magnolia hookeri & & & $\mathrm{P}$ & & \\
\hline 13. Magnolia insignis & $\mathrm{P}$ & & $\mathrm{P}$ & & \\
\hline 14. Magnolia kingii & & $\mathrm{P}$ & $\mathrm{P}$ & $\mathrm{P}$ & \\
\hline 15. Magnolia kisopa & $\mathrm{P}$ & $\mathrm{P}$ & $\mathrm{P}$ & & \\
\hline 16. Magnolia lanuginosa & $\mathrm{P}$ & $\mathrm{P}$ & $\mathrm{P}$ & & \\
\hline 17. Magnolia liliifera & & $\mathrm{P}$ & $\mathrm{P}$ & & \\
\hline 18. Magnolia mannii & & & $\mathrm{P}$ & $\mathrm{P}$ & \\
\hline 19. Magnolia nilagirica & & & $\mathrm{P}$ & & $\mathrm{P}$ \\
\hline 20. Magnolia oblonga & & & $\mathrm{P}$ & $\mathrm{P}$ & \\
\hline 21. Magnolia pealiana & & & $\mathrm{P}$ & & \\
\hline 22. Magnolia pleiocarpa & & & $\mathrm{P}$ & & \\
\hline 23. Magnolia pterocarpa & $\mathrm{P}$ & $\mathrm{P}$ & $\mathrm{P}$ & $\mathrm{P}$ & \\
\hline 24. Magnolia punduana & & & $\mathrm{P}$ & & \\
\hline 25. Magnolia rabaniana & & $\mathrm{P}$ & $\mathrm{P}$ & & \\
\hline
\end{tabular}


Uma Shankar 147

\begin{tabular}{|l|c|c|c|c|c|}
\hline Taxon name & Nepal & Bhutan & India & Bangladesh & $\begin{array}{c}\text { Sri } \\
\text { Lanka }\end{array}$ \\
\hline Introduced and hybrid taxa & & & & & \\
\hline 26. Magnolia $\times$ alba & & & $\mathrm{P}$ & & \\
\hline 27. Magnolia coco & & & $\mathrm{P}$ & & \\
\hline 28. Magnolia grandiflora & $\mathrm{P}$ & $\mathrm{P}$ & $\mathrm{P}$ & & \\
\hline 29. Magnolia figo var. figo & & & $\mathrm{P}$ & & \\
\hline 30. Magnolia liliiflora & $\mathrm{P}$ & $\mathrm{P}$ & $\mathrm{P}$ & & \\
\hline 31. Magnolia montana & & & $\mathrm{P}$ & & \\
\hline 32.Magnolia obovata & & & $\mathrm{P}$ & & \\
\hline 33.Magnolia sirindhorniae & & & $\mathrm{P}$ & & \\
\hline 34. Magnolia $\times$ soulangeana & & & $\mathrm{P}$ & & \\
\hline Total taxa & 10 & 13 & 34 & 10 & 1 \\
\hline
\end{tabular}

[Note: The states of Pakistan and Maldives do not have species from Magnoliaceae; P, present]

In northeast India, magnolias are confined to the horse-shoe shaped mountainous terrain encircling the Assam Valley involving the States of Arunachal Pradesh, Nagaland, Manipur, Mizoram, Meghalaya and foot hills of Assam (Table 4). Assam tops the list in terms of presence of maximum number of magnolias (26 taxa), which is closely followed by Arunachal Pradesh (21 taxa). Manipur, Meghalaya and Nagaland have 15, 13 and 11 taxa respectively. Mizoram and Sikkim has eight taxa each. A few sporadic occurrences of magnolias are reported from Tripura. Seven native species, viz., M. campbellii, M. cathcartii, M. champaca, M. doltsopa, M. hodgsonii, M. kisopa, and M. lanuginosa occur in Darjeeling district of West Bengal which is an adjoining territory to the state of Sikkim in East Himalaya. In addition, introduced species like M. coco, Magnolia figo, M. grandiflora and M. liliiflora are also growing in Darjeeling Terai and Hills (Das \& Chanda 1987; Bhujel 1996; Das \& Ghosh 2009). Outside northeast India, M. liliifera is reported from Andaman \& Nicobar Islands, although $M$. champaca is introduced there. M. nilagirica is the only magnolia present in Western Ghats. Rare occurrences of $M$. champaca and M. hodsonii are known from the foothills of central Himalayas (Table 4).

Table 4. Distribution of Magnoliaceae in different States of India based on the reports in regional floras.

\begin{tabular}{|l|c|c|c|c|c|c|c|c|c|c|c|}
\hline \multicolumn{1}{|c|}{ Taxon name } & AR & AS & MN & ML & MZ & NL & SK & TR & WB & AN & IN \\
\hline Native taxa & & & & & & & & & & & \\
\hline 1. Magnolia baillonii & $\mathrm{P}$ & $\mathrm{P}$ & & $\mathrm{P}$ & $\mathrm{P}$ & & & $\mathrm{P}$ & & & \\
\hline 2. Magnolia campbellii & $\mathrm{P}$ & $\mathrm{P}$ & $\mathrm{P}$ & & & $\mathrm{P}$ & $\mathrm{P}$ & & $\mathrm{P}$ & & \\
\hline 3. Magnolia cathcartii & & $\mathrm{P}$ & $\mathrm{P}$ & & & $\mathrm{P}$ & $\mathrm{P}$ & & $\mathrm{P}$ & & \\
\hline 4. Magnolia caveana & $\mathrm{P}$ & $\mathrm{P}$ & & $\mathrm{P}$ & & & & & & & \\
\hline 5. Magnolia champaca & $\mathrm{P}$ & $\mathrm{P}$ & $\mathrm{P}$ & $\mathrm{P}$ & & $\mathrm{P}$ & & $\mathrm{P}$ & $\mathrm{P}$ & & $\mathrm{P}$ \\
\hline 6.Magnolia champaca var. champaca & & & & $\mathrm{P}$ & $\mathrm{P}$ & & & & & & \\
\hline 7. Magnolia doltsopa & $\mathrm{P}$ & $\mathrm{P}$ & $\mathrm{P}$ & $\mathrm{P}$ & $\mathrm{P}$ & $\mathrm{P}$ & & $\mathrm{P}$ & $\mathrm{P}$ & & \\
\hline 8. Magnolia globosa & $\mathrm{P}$ & $\mathrm{P}$ & & & & & & & $\mathrm{P}$ & & \\
\hline 9. Magnolia griffithii & $\mathrm{P}$ & $\mathrm{P}$ & $\mathrm{P}$ & & & $\mathrm{P}$ & & & & & \\
\hline 10. Magnolia gustavi & $\mathrm{P}$ & $\mathrm{P}$ & & & & & & & & & \\
\hline 11. Magnolia hodgsonii & $\mathrm{P}$ & $\mathrm{P}$ & $\mathrm{P}$ & $\mathrm{P}$ & $\mathrm{P}$ & & $\mathrm{P}$ & & $\mathrm{P}$ & & $\mathrm{P}$ \\
\hline 12. Magnolia hookeri & $\mathrm{P}$ & $\mathrm{P}$ & $\mathrm{P}$ & & & & & & & & \\
\hline 13. Magnolia insignis & $\mathrm{P}$ & $\mathrm{P}$ & $\mathrm{P}$ & $\mathrm{P}$ & & $\mathrm{P}$ & & & & & \\
\hline 14. Magnolia kingii & $\mathrm{P}$ & $\mathrm{P}$ & $\mathrm{P}$ & $\mathrm{P}$ & & & $\mathrm{P}$ & & & & \\
\hline 15. Magnolia kisopa & $\mathrm{P}$ & & $\mathrm{P}$ & & & $\mathrm{P}$ & $\mathrm{P}$ & & $\mathrm{P}$ & & \\
\hline 16. Magnolia lanuginosa & $\mathrm{P}$ & $\mathrm{P}$ & $\mathrm{P}$ & & & $\mathrm{P}$ & & & $\mathrm{P}$ & & \\
\hline 17. Magnolia liliifera & & $\mathrm{P}$ & & & & & $\mathrm{P}$ & $\mathrm{P}$ & & $\mathrm{P}$ & \\
\hline
\end{tabular}




\begin{tabular}{|l|c|c|c|c|c|c|c|c|c|c|c|}
\hline \multicolumn{1}{|c|}{ Taxon name } & AR & AS & MN & ML & MZ & NL & SK & TR & WB & AN & IN \\
\hline 18. Magnolia mannii & $\mathrm{P}$ & $\mathrm{P}$ & & & & & & & & & \\
\hline 19. Magnolia nilagirica & & $\mathrm{P}$ & & & & & & & & & $\mathrm{P}$ \\
\hline 20. Magnolia oblonga & $\mathrm{P}$ & $\mathrm{P}$ & & $\mathrm{P}$ & $\mathrm{P}$ & $\mathrm{P}$ & & & & & \\
\hline 21. Magnolia pealiana & $\mathrm{P}$ & $\mathrm{P}$ & & & & & & & & & \\
\hline 22. Magnolia pleiocarpa & & $\mathrm{P}$ & & & & & & & & & \\
\hline 23. Magnolia pterocarpa & $\mathrm{P}$ & $\mathrm{P}$ & & $\mathrm{P}$ & & & & $\mathrm{P}$ & & & \\
\hline 24. Magnolia punduana & $\mathrm{P} *$ & & $\mathrm{P} *$ & $\mathrm{P}$ & & & & & & & \\
\hline 25. Magnolia rabaniana & $\mathrm{P}$ & $\mathrm{P}$ & & $\mathrm{P}$ & $\mathrm{P}$ & $\mathrm{P}$ & & & & & \\
\hline Introduced and hybrid taxa & & & & & & & & & & & \\
\hline 26. Magnolia x alba & & & $\mathrm{P}$ & & & & & & & & \\
\hline 27. Magnolia coco & & & $\mathrm{P}$ & & & & & & $\mathrm{P}$ & & \\
\hline 28. Magnolia grandiflora & $\mathrm{P}$ & $\mathrm{P}$ & $\mathrm{P}$ & $\mathrm{P}$ & $\mathrm{P}$ & $\mathrm{P}$ & $\mathrm{P}$ & $\mathrm{P}$ & $\mathrm{P}$ & $\mathrm{P}$ & $\mathrm{P}$ \\
\hline 29. Magnolia figo var. figo & & $\mathrm{P}$ & & & & & & & $\mathrm{P}$ & & \\
\hline 30. Magnolia liliiflora & & & & & & & $\mathrm{P}$ & & $\mathrm{P}$ & & \\
\hline 31. Magnolia montana & & $\mathrm{P}$ & & & $\mathrm{P}$ & & & $\mathrm{P}$ & & & \\
\hline 32.Magnolia obovata & & $\mathrm{P}$ & & & & & & & & & \\
\hline 33.Magnolia sirindhorniae & & & & & & & & & & & $\mathrm{P}$ \\
\hline 34. Magnolia $\times$ soulangeana & & & & & & & & & $\mathrm{P}$ & & $\mathrm{P}$ \\
\hline Total taxa & 21 & 26 & 15 & 13 & 8 & 11 & 8 & 7 & 13 & 2 & 6 \\
\hline
\end{tabular}

[Acronyms: AR, Arunachal Pradesh; AS, Assam; MN, Manipur; ML, Meghalaya; MZ, Mizoram; NL, Nagaland; SK, Sikkim; TR, Tripura; WB, West Bengal (mainly Darjeeling district); AN, Andaman \& Nicobar Islands; IN, rest of India; P, present; *, doubtful]

The magnolias in northeast India occur mostly in tropical and subtropical rainforests and their abundances are moderate to low, signifying their rarity (Shankar \& Tripathi 2017; Shankar 2017, 2019). For instance, in tropical rainforests of Meghalaya, M. hodgsonii, M. champaca, and M. insignis were encountered with densities of 5.3, 1.2 and 0.4 stems ha ${ }^{-1}$, respectively (Shankar \& Tripathi 2017). In these forests, the dominant associates were Boebmeria glomerulifera, Castanopsis armata, Cinnamomum tamala, Itea macrophylla, Macropanax undulatus, Oreocnide integrifolia, Persea odoratissima, Schima wallichii and Sysygium tetragonum. In Shorea robusta-dominated forests of eastern India, $M$. champaca and $M$. hodgsonii are common magnolias, but with low densities. For instance, $M$. champaca with 3 stems $\mathrm{ha}^{-1}$ and $M$. hodgsonii with 0.5 stems ha ${ }^{-1}$ occurred in Mahananda Wildlife Sanctuary in Darjeeling (Shankar 2001). Here, the associate species were Amoora rohituka, Cryptocarya floribunda, Dillenia pentagyna, Leea spp., Mallotus philippensis, Schima wallichii, and Terminalia belerica (Shankar 2001). In 'Khasi Hill Sal' forest ecosystem in Meghalaya, M. champaca occurred with a density of 0.2 stems ha $^{-1}$ (Tripathi \& Shankar 2014). These results indicate that the magnolias are rare in natural forests and their populations are threatened.

\section{CONCLUSIONS}

The fossil and historical records reveal that the magnolias have lived and adapted to very diverse climates, i.e., from very cold to very warm. The magnolias were once dominant in the northern hemisphere (Heywood 1978), but descended towards south and are now predominant in southeast Asia as a result of changes in the climate. The deciduous, temperate species are derived from evergreen, warm-temperate species as an adaptation to the climate (Hebda \& Irving 2004). In general, the magnolias have lost their dominance in most habitats, as also found in this study. Once dominant in high latitudes of northern hemisphere, magnolias now remain plants of occasional or rare occurrence in many habitations. The unfolding of complex global climate change scenario due to exploding human population pressure poses enormous uncertainty for future as to how will the remaining species and their assemblages respond to maintaining or altering their populations and distributions in both protected and distressed environments. 


\section{Acknowledgements}

The author is honoured to dedicate this article to Dr. R.R. Rao, FNA who founded teaching and research of plant taxonomy at NEHU, Shillong in 1970s and began publishing a botanical magazine entitled "Magnolia". The author acknowledges the facilities afforded at the Department of Botany, North-Eastern Hill University, Shillong where he is studying the patterns in the forests and the flora of northeastern region of India under the doctor of science programme. The author expresses gratitude to Shilpi Agrawal and Amogha Shankar for help in preparation of this manuscript and gratefully thanks the authorities of the Botanical Survey of India for access to herbarium.

\section{LITERATURE CITED}

APG IV 2016. An update of the Angiosperm Phylogeny Group classification for the orders and families of flowering plants: APG IV. Bot. J. Linn. Soc. 181: 1 - 20.

Balakrishnan, N.P. 1981 \& 1983. Flora of Jowai and vicinity. Vols. I, II. Botanical Survey of India, Howrah.

Barooah, C. \& Ahmed, I. 2014. Plant diversity of Assam- a checklist of angiosperms \& gymnosperms. Assam Science Technology and Environment Council, Assam.

Bhujel, R.B. 1996. Studies on the Dicotyledonous Flora of Darjeeling District. Ph.D. thesis, University of North Bengal, Siliguri.

Chowdhury, S. 2005. Assam's flora - present status of vascular plants. Assam Science Technology and Environment Council, Guwahati, Assam.

Cicuzza, D.; Newton, A. \& Oldfield, S. 2007. The Red List of Magnoliaceae. Fauna \& Flora International, Cambridge, UK.

Das, A.P. \& Chanda, S. 1987. Flowering calendar of the angiospermic flora of Darjeeling Hills, West Bengal (India). Trans. Bose Res. Inst. 51(4): 99 - 133.

Das, A.P. \& Ghosh, C. 2009. Germplasm Collection in the Garden of Medicinal Plants, University of North Bengal. University of North Bengal, Siliguri.

Deb, D.B. 1981 \& 1983. The flora of Tripura State. Vols. I \& II. Today \& Tomorrow's Printers and Publishers, New Delhi.

Doyle, J.A. 2012. Molecular and fossil evidence on the origin of angiosperms. Annu. Rev. Earth Planet. Sci. 40: $301-326$.

Doyle, J.A. 2014. Plant evolution. AccessScience from McGraw Hill Education 4: 1 - 6.

Every, J.L.R. \& Baracat, A. 2009 onwards. Neotropical magnoliaceae. In: Milliken, W., Klitgård, B. \& Baracat, A. (eds.), Neotropikey - Interactive key and information resources for flowering plants of the Neotropics. (http://www.kew.org/science/tropamerica/neotropikey/families/ Magnoliaceae.htm)

Figlar, R.B. \& Nooteboom, H.P. 2004. Notes on Magnoliaceae IV. Blumea 49(1): 87 - 100.

Friis, E.M.; Crane, P.R. \& Pedersen, K.R. 2011. Early Fowers and Angiosperm Evolution. Cambridge University Press.

Frohlich, M.W. \& Chase, M.W. 2007. After a dozen years of progress the origin of angiosperms is still a great mystery. Nature 450: $1184-1189$.

Giri, G.S.; Pramanik, A. \& Chowdhery, H.J. 2008. Materials for the flora of Arunachal Pradesh. Vol. II. Asteraceae-Ceratophyllaceae. Botanical Survey of India, Calcutta. 
Global Tree Specialist Group 2014. Magnolia pleiocarpa. The IUCN Red List of Threatened Species 2014: e.T193991A2293552. https://dx.doi.org/10.2305/IUCN.UK.20141.RLTS.T193991A2293552.en. Accessed on 26 June 2020.

Hajra, P.K.; Verma, D.M. \& Giri, G.S. 1996. Materials for the flora of Arunachal Pradesh. Vol. I. Ranunculaceae-Dipsacaceae. Botanical Survey of India, Calcutta.

Haridasan, K. \& Rao, R.R. 1985 \& 1987. Forest flora of Meghalaya. Vols. I, II. Botanical Survey of India, Howrah.

Hebda, R.J. \& Irving, E. 2004. Origin and History of Magnolias. American Geophysical Union, Fall Meeting 2004, abstract id. U31B-07.

Heywood, V.H. 1978. Flowering Plants of the World. Oxford University Press, Oxford.

Hooker, J.D. 1872 - 1897. The Flora of British India. Vols. 1 - 7. L. Reeve and Co., Ashford, Kent, London.

Iralu, V.; Pao, N.T. \& Upadhaya, K. 2020. An assessment of population structure and regeneration status of Magnolia punduana Hk. f. \& Th. (Magnoliaceae) in fragmented forests of northeast India. J. For. Res. 31(3): 937 - 943.

Joseph, J. 1982. Flora of Nongpoh and its vicinity. Botanical Survey of India, Howrah.

Kanjilal, U.N.; Kanjilal, P.C.; Das, A. \& Purkayastha, C. 1934. Flora of Assam, Vol. 1. Government of Assam, Shillong.

Khuraijam, J.S. \& Goel, A.K. 2015. Enumeration of the genus Magnolia L. in India with its conservation status. Phytotaxonomy 15: 107 - 113.

Kundu, S.R. 2009. A synopsis on distribution and endemism of Magnoliaceae s.l. in Indian Subcontinent. Thaiszia - J. Bot., Košice 19: 47 - 60.

Mao, A.A.; Sinha, B.K..; Verma, D. \& Sarma, N. 2016. Checklist of flora of Meghalaya. Botanical Survey of India, Kolkata.

Mao, A.A.; Odyuo, N.; Verma, D. \& Singh, P. 2017. Checklist of flora of Nagaland. Botanical Survey of India, Kolkata.

Nooteboom, H.P. 2011. How did magnolias (Magnoliaceae, Magnolioideae) reach tropical Asia. Proceedings of the Second International Symposium on the family Magnoliaceae. Huazhong University Science Technical Press. Pp. 22 - 29.

POWO 2020. Plants of the World Online. Kew Science, UK. Accessed on May 20, 2020.

Sanchez-Velasquez, L.R.; Pineda-Lopez, M. del R.; Vasquez-Morales, S.G. \& AvendanoYanez, M.L. 2016. Ecology and Conservation of Endangered Species: The Case of Magnolias. In. Quinn, M. (ed.), Endangered species. Nova Science Publishers, Inc. Pp. $63-84$.

Sauquet, H. et al. 2017. The ancestral flower of angiosperms and its early diversification. Nature Communications 8: 16047. DOI: 10.1038/ncomms16047.

Shankar, U. 2001. A case of high tree diversity in a 'sal' (Shorea robusta)-dominated lowland forest of eastern Himalaya: floristic composition, regeneration and conservation. Curr. Sci. 81(7): $776-786$.

Shankar, U. 2017. Yes, we have tropical rainforest far from the equator in Meghalaya. The NEHU J. 15(1): 1 - 9.

Shankar, U. 2019. Phytosociology of stratification in a lowland tropical rainforest occurring north of the tropic of cancer in Meghalaya, India, Pl. Diver. 41: 295 - 299. 
Shankar, U. \& Tripathi, A.K. 2017. Rainforests north of the Tropic of Cancer: physiognomy, floristics and diversity in 'lowland rainforests' of Meghalaya, India. Pl. Diver. 39: 20 -36 .

Singh, N.P.; Chauhan, A.S. \& Mondal, A.S. 2000. Flora of Manipur. Vol. I. Botanical Survey of India, Howrah.

Singh, N.P., Singh, K.P. \& Singh, D.K. 2002. Flora of Mizoram. Vol. I. Botanical Survey of India, Howrah. pp. 845.

The Plant List 2013. The Plant List. Version 1.1. http://www.theplantlist.org/. [Accessed on: June 19, 2020]

Tripathi, A.K. \& Shankar, U. 2014. Patterns of species dominance, diversity and dispersion in 'Khasi hill sal' forest ecosystem in northeast India. For. Ecosys. 1: 1-20.

www.plantsoftheworldonline.org 